\title{
The Impact of Nuclear Physics during Stellar Core Collapse
}

\author{
W. Raphael Hix ${ }^{* a b}$ Eric J. Lentz, ${ }^{b a}$ Mark L. Baird, ${ }^{b a}$ O.E. Bronson Messer, ${ }^{c b}$ and \\ Anthony Mezzacappa ${ }^{a b}$ \\ ${ }^{a}$ Physics Division, Oak Ridge National Laboratory \\ Oak Ridge, TN 37831 \\ ${ }^{b}$ Department of Physics \& Astronomy, University of Tennessee \\ Knoxville, TN 37996 \\ ${ }^{c}$ National Center for Computational Science, Oak Ridge National Laboratory \\ Oak Ridge, TN 37831 \\ E-mail: raph@ornl.gov
}

\begin{abstract}
Nuclear electron capture and the nuclear equation of state play important roles during the collapse of a massive star and the subsequent supernova. The nuclear equation of state controls the nature of the bounce which initially forms the supernova shock while electron capture determines the location where the shock forms. Advances in nuclear structure theory have allowed a more realistic treatment of electron capture on heavy nuclei to be developed. We will review how this improvement has led to a change in our understanding of stellar core collapse, with electron capture on nuclei with masses larger than 50 found to dominate electron capture on free protons, resulting is significant changes in the hydrodynamics of core collapse and bounce. We will also demonstrate the impact of a variety of nuclear equations of state on supernova shock propagation. Of particular note is the interplay between the nuclear composition determined by the equation of state and nuclear electron capture.
\end{abstract}

10th Symposium on Nuclei in the Cosmos

July 27 - August 12008

Mackinac Island, Michigan, USA

\footnotetext{
*Speaker.
} 


\section{Nuclear Inputs for Stellar Core Collapse}

The end of a massive ( $\gtrsim 10 M_{\odot}$ ) star's life begins with central silicon burning and the formation of the iron core. Composed of maximally-bound iron and neighboring nuclei, the iron core is unable to tap thermonuclear fusion to slow the inexorable contraction that results from the star's self gravity. Continued silicon burning at the outer edge of this cold iron core causes it to grow, until it is too massive to be supported by the pressure of degenerate electrons. Core collapse then ensues, subsonically in the homologous inner region of the core, supersonically in its outer regions. During collapse, increasing density allows electron captures on heavy nuclei and protons to deleptonize the matter, accelerating the collapse. When the inner core exceeds nuclear densities, it becomes incompressible. The suddenly stiffened inner core, now a proto-neutron star (PNS), rebounds, colliding with the supersonically infalling outer core, producing a shock that initially drives these layers outward. Though this shock is stalled by neutrino losses and energy lost dissociating the heavy nuclei, it is ultimately revived, most likely by tapping the large flux of neutrinos that are carrying away the binding energy of the newly formed PNS. While much of the physics that is important during this latter reheating phase is dominated by dissociated nucleons, the low entropy during collapse results in a composition dominated by heavy nuclei [1]. As we will describe in later sections, this allows nuclear physics to have a strong impact on the behavior of core collapse, setting the stage for the supernova explosion. This impact is dominated by two factors, the nuclear equation of state and nuclear interactions with leptons.

\section{Nuclear Interactions with Electrons and Neutrinos}

Improvements in nuclear structure calculations in recent years have allowed re-examination of the role of nuclear interactions with electrons and neutrinos. Unlike prior work [2, 3], based on the independent particle model (IPM), which suppressed these interactions once the $0 f_{5 / 2}$ neutron shell closed when the neutron number exceeded 40, modern calculations reveal continued electron capture reactions throughout collapse [4]. The result has been a new appreciation of the dominant role of nuclear electron capture during core collapse [5]. Figure 1 illustrates the differences that continued nuclear electron capture cause as the inner core bounces. The LMSH prescription is based on the recent shell model [6] and SMMC+RPA [7] rates, the Bruen85 prescription [3] on the older IPM rates. General Relativity is considered in these Agile-Boltztran [8] simulations which use opacities from Ref. [3], aside from the nuclear electron capture. Clearly visible is a deeper launching point for the supernova shock, indicating a $\sim 20 \%$ smaller initial mass of the proton neutron star, the result of the additional deleptonization in the core due to capture on nuclei with $\mathrm{N}>40$. In the outer layers $\left(0.6-1.1 M_{\odot}\right)$, deleptonization is slower with recent shell model rates for nuclei with $A \sim 60$ [6] than with the prior (IPM) rates, resulting in slower collapse of these layers. During the reheating phase, this results in lower density and velocity in the infalling layers and thus less opposition to the shocks attempts to move outward.

More recently, the impact of similarly improved rates for inelastic neutrino-nucleus scattering (INNS) [9] have been considered by Langanke et al. [10]. The only prior examination of INNS [11] used a rate for scattering on ${ }^{56} \mathrm{Fe}$ based on a zero temperature nuclear model combining a truncated nuclear shell model evaluation of the allowed contributions with Goldhaber-Teller estimates for 


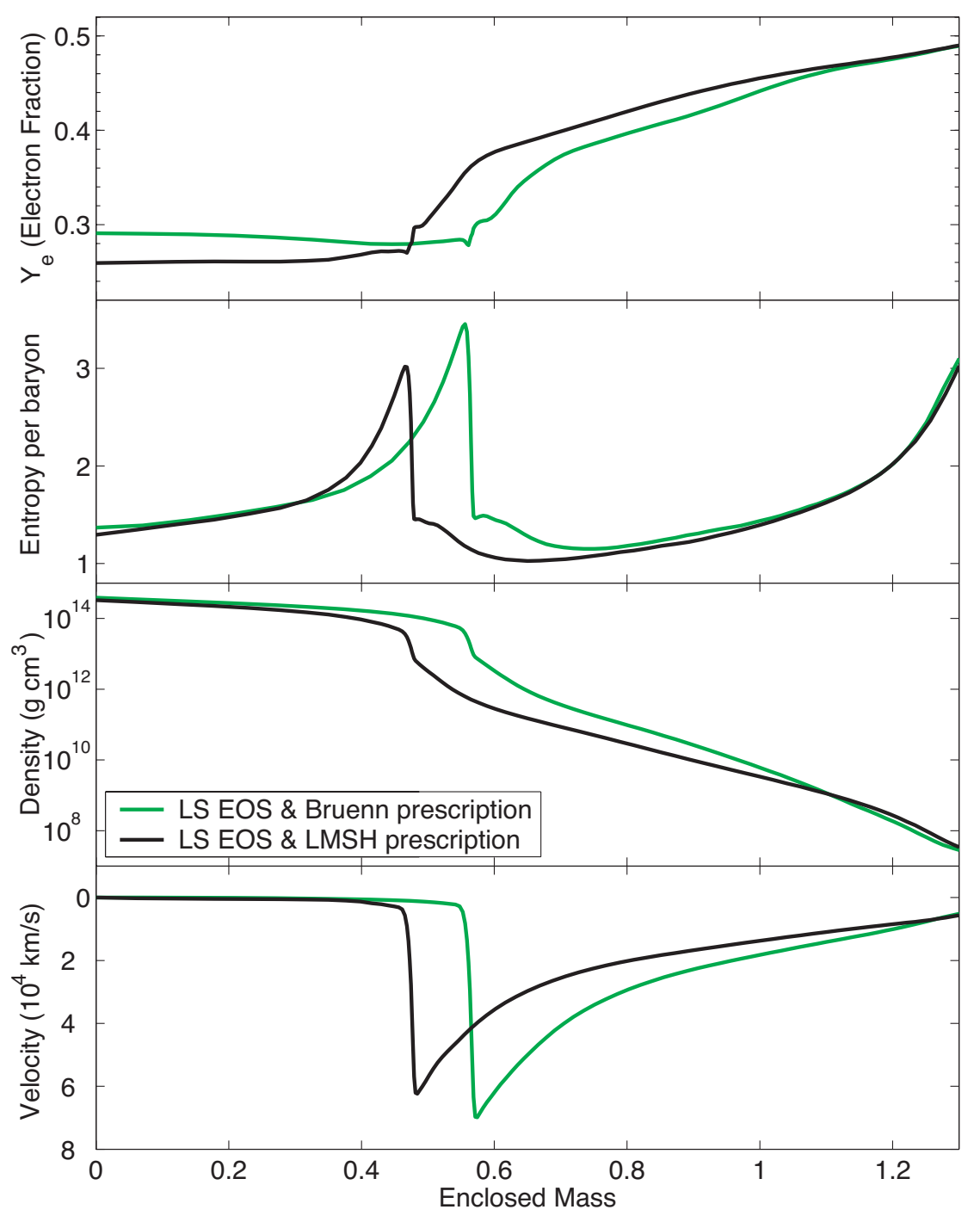

Figure 1: Effects of nuclear electron capture at bounce for a $15 M_{\odot}$ progenitor [5]. The black lines illustrates simulations using recently updated rates for nuclear electron capture, the red lines illustrate simulations using older IPM rates.

forbidden contribution and found INNS to be competitive during collapse with neutrino electron scattering in equilibrating the neutrinos with the matter. While newer simulations confirmed this, only negligible differences are seen in the supernova dynamics as neutrino electron scattering is already quite efficient at equilibrating the neutrinos and matter. Small regions do experience significant additional heating due to down-scattering in energy of neutrinos, but this occurs only where the large neutrino flux of the breakout burst encounters unshocked iron and thus is highly localized in space and time. Furthermore this matter is falling supersonically toward the shock, thus additional neutrino heating has little dynamical impact. However, this down-scattering of the neutrinos does significantly suppress the high energy tail of the neutrino distribution, potentially impacting 
our terrestrial neutrino detection capability [10].

\section{Equations of State}

In general, the equation of state (EoS) is a thermodynamic relation between the pressure, temperature and internal energy, providing a closure to the hydrodynamic equations. For stellar matter, contributions from electrons/positrons, photons and atomic nuclei all must be considered. The dominance of the maximally bound, iron-peak nuclei in the iron core is the result of thermodynamic equilibrium for strong and electromagnetic nuclear reactions, termed Nuclear Statistical Equilibrium (NSE). During collapse, the increasing density and neutronization causes NSE to favor heavier, more neutron-rich nuclei. This leads eventually to a composition dominated by exotic columnar and planar nuclear states [12] and ultimately, when the core reaches densities similar to those of the nucleons in a nucleus, nuclear matter. The development of NSE makes the nuclear composition a function of the thermodynamic state and electron fraction $\left(Y_{e}\right)$, allowing the composition to be evolved as part of the equation of state, unlike concentric layers of successively lighter elements that lie above the iron core, whose evolution must be followed with a nuclear reaction network. Ultimately, all of these phases of baryonic matter must be included in the supernova EoS.

The thermodynamic state and composition in the core naturally has a profound impact on the hydrodynamic evolution and neutrino radiation transport in core-collapse supernovae. For example, the matter pressure, as determined by the thermodynamic state, causes the bounce and drives much of the hydrodynamic motion, while the composition of the matter has a great effect on the neutrino opacities, determining how effectively energy is transfered from the neutrino radiation field to the matter. For these reasons, considerable effort has been invested in supernova equations of state. Here we will compare three, those produced by Lattimer and Swesty [13], Shen et al. [14] and Wilson and Mathews [15]. In addition to thermodynamic variables like pressure, internal energy and entropy, all three of these EoSs provide compositional data in the form of the mass fractions of protons, neutrons, $\alpha$-particles and a representative or average heavy nucleus, along with the atomic number and mass of this average heavy nucleus.

The equation of state of Lattimer \& Swesty [13] (LS) has long been the staple EoS for supernova simulations. It is based on a compressible liquid drop model, similar to that of Lamb et al. [16], but in a lightweight form suitable for calculation within a supernova model. The provided FORTRAN routines calculate the EoS constituents for photons, electrons/positrons, nuclei and nuclear matter. The nuclear composition for non-uniform nuclear matter is calculated in the Wigner-Seitz approximation of a heavy nucleus in a vapor of nucleons and $\alpha$-particles. The LS EoS assumes a nuclear saturation density $\left(\rho_{s}\right)$ of $2.59 \times 10^{14} \mathrm{~g} \mathrm{~cm}^{-3}$, a symmetry energy $\left(E_{\text {sym }}\right)$ of $29.3 \mathrm{MeV}$ and allows a selection of the nuclear incompressibility $\left(\kappa_{s}\right)$. For consistency with our earlier work, the simulations described here use $\kappa_{s}=180 \mathrm{MeV}$, but simulations using a more realistic $\kappa_{s}=220 \mathrm{MeV}$ are similar.

The equation of state of Shen, Toki, Oyamatsu \& Sumiyoshi [14] (STOS) is based on the relativistic mean field theory. It is constructed assuming $\rho_{s}=2.43 \times 10^{14} \mathrm{~g} \mathrm{~cm}^{-3}, E_{\text {sym }}=36.9 \mathrm{MeV}$ and $\kappa_{s}=281 \mathrm{MeV}$. Thomas-Fermi calculations [17] provide the nuclear composition for nonuniform nuclear matter, also using the Wigner-Seitz approximation. The EoS is provided in tabular 


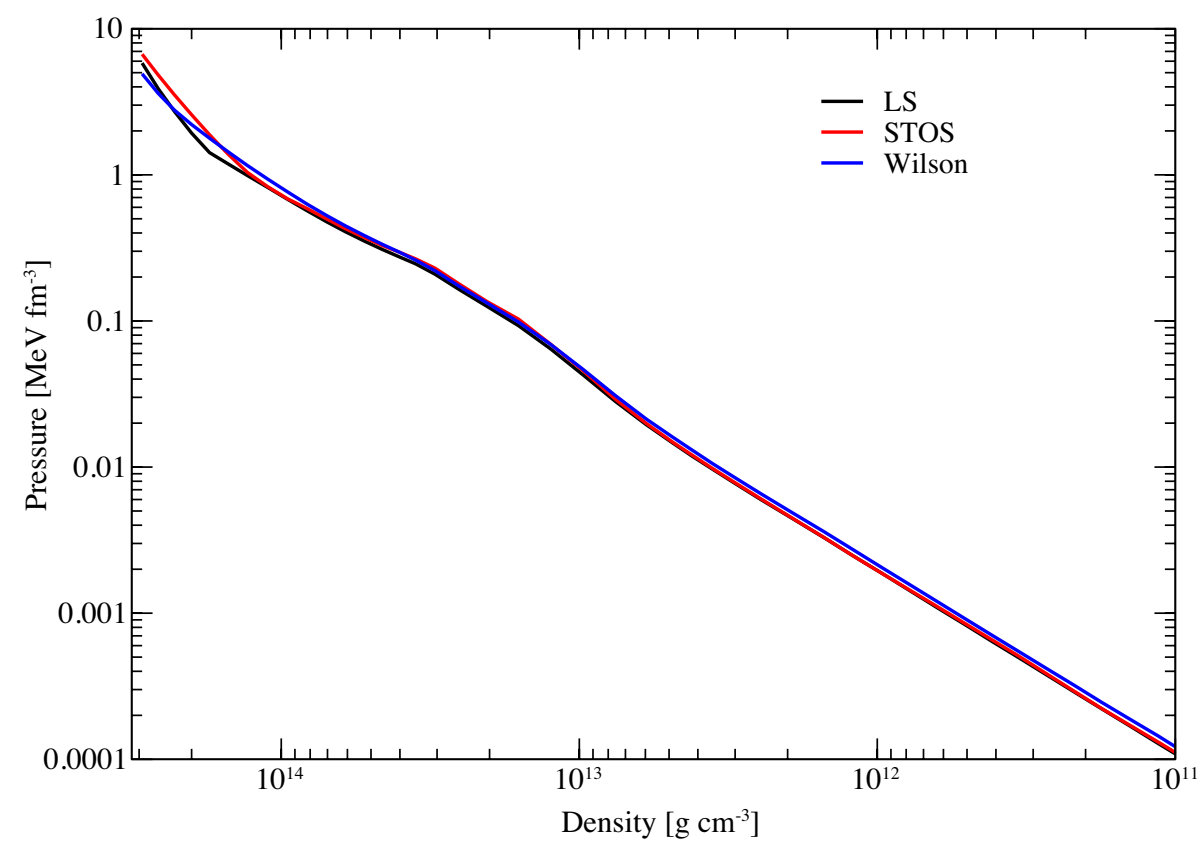

Figure 2: Comparison of Pressure calculated by the EoS under identical conditions. The black, red and blue lines illustrate results from simulations using the EoS of Lattimer and Swesty [13], Shen et al. [14] and Wilson and Mathews [15], respectively.

form, including only the nuclear contributions. To implement this EoS in supernova simulations, we have employed the photon and electron/positron contributions from the LS EoS.

The equation of state of Wilson and his collaborators is described most fully in Wilson and Mathews [15] (see also [18-20]). For supranuclear matter, the empirical prescription of Baron et al. [21] is used with $\rho_{s}=2.66 \times 10^{14} \mathrm{~g} \mathrm{~cm}^{-3}, \kappa_{s}=200 \mathrm{MeV}$ and the supranuclear adiabatic index, $\gamma_{s},=2.75$. These choices for $\rho_{s}, \kappa_{s}$ and $\gamma_{s}$, as well as the form of the symmetry energy $E_{\text {sym }}=16\left(1-2 Y_{e}\right)^{2}\left(\rho / \rho_{s}\right)\left(1+4.5 /\left(1+4 \rho / \rho_{s}\right)\right) \mathrm{MeV}$, were informed by the Relativistic Brueckner-Hartree-Fock calculations of [22]. The Wilson EoS also includes the effects of pion production at high density, using the model of Friedman et al. [23]. This model is constrained by comparision between experimental measurements and simulations of pion production in heavy ion collisions [20]. The Wilson EoS was provided to us as a table by S. Dalhead at LLNL. The table included contributions from photons, electrons/positrons and baryons, but did not provide the chemical potentials for electrons, protons and neutrons. For our simulations, the electron chemical potential is taken from LS EoS while the proton and neutron chemical potentials are calculated analytically from their tabulated abundances. The identity of the average heavy nucleus is calculated from a density and electron fraction dependent analytic formula [15].

\subsection{Comparison under Consistent Conditions}

Comparison of the three equations for state considered here under identical, pre-calculated conditions is very informative. Figures $2 \& 3$ compare the pressure and composition for all three EoSs as a function of density, using the temperature, density and $Y_{e}$ conditions for a collapsing 


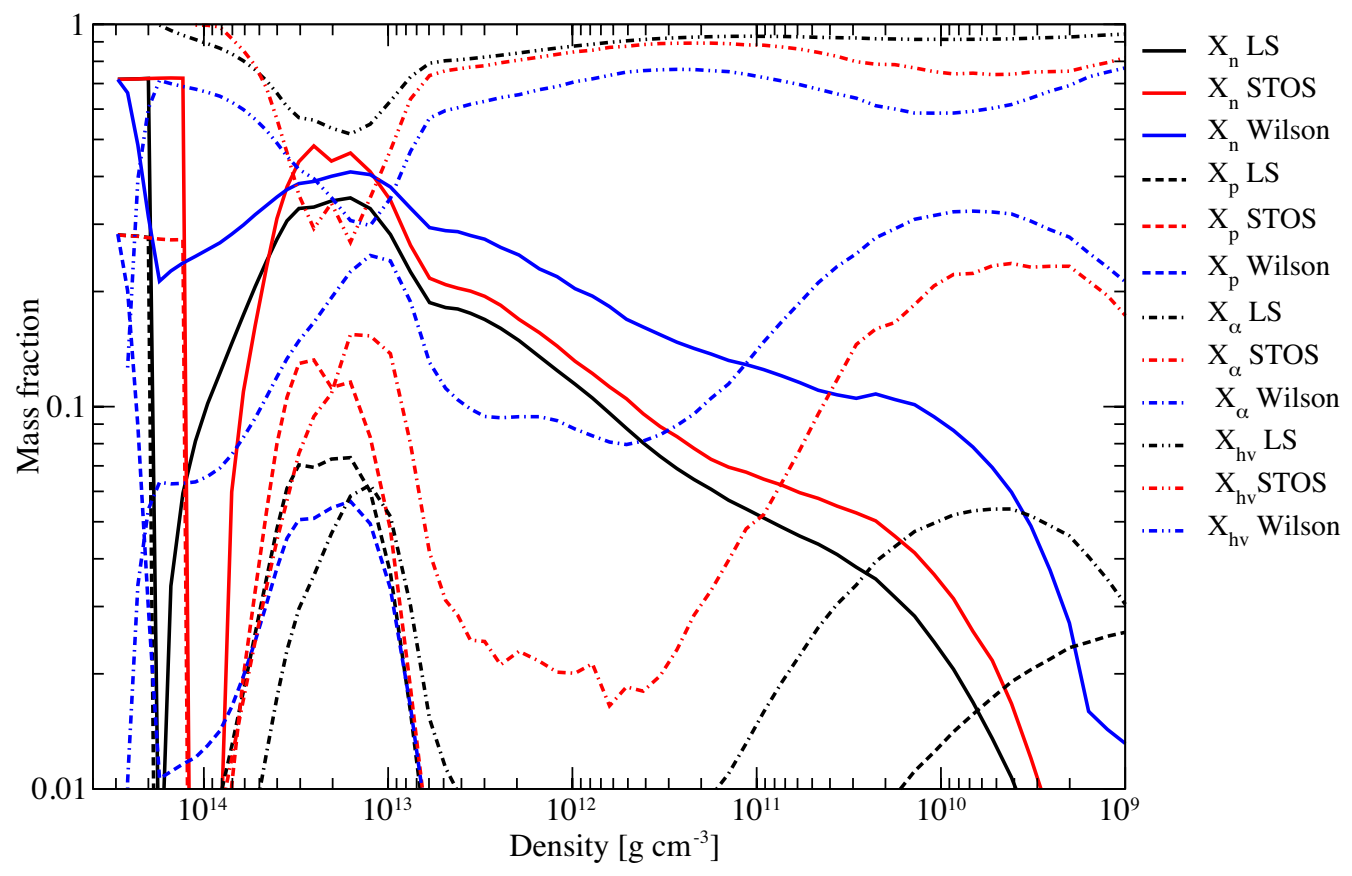

Figure 3: Comparison of Nuclear composition calculated by the EoS under identical conditions. The black, red and blue lines illustrate results from simulations using the EoS of Lattimer and Swesty [13], Shen et al. [14] and Wilson and Mathews [15], respectively.

$15 M_{\odot}$ star just before bounce, from the simulation using the LMSH prescription and LS EoS presented in Figure 1. The pressure shows very small differences in the subnuclear regime, where it is dominated by the degenerate electron contribution, but even at supranuclear densities, only $\sim 30 \%$ variations are seen. STOS EoS exhibits the highest supranuclear pressures, consistent with its larger incompressibility, while the Wilson EoS's pressure is moderately larger than that of the LS EoS for densities below saturation, but lower at the highest densities. In contrast to the limited extent of differences in the pressure, variations of $\sim 30 \%$ are seen in the dominant mass fractions throughout the star, with even larger variations in the smaller mass fractions. These differences are seen even at relatively low density where the average heavy nuclei are well understood species. These differences are also reflected in the entropy, which is dominated by the nuclear contribution.

\subsection{Collapse Simulation Results}

Full simulations were run with each of the three EoSs, using Agile-Boltztran. These simulations include General Relativity, which is important for a nuclear EoS comparison since the inclusion of GR results in roughly $30 \%$ higher central densities thus better exercising the supranuclear portion of the EoS. To facilitate comparison with the results of Hix et al. [5], neutrino opacities 


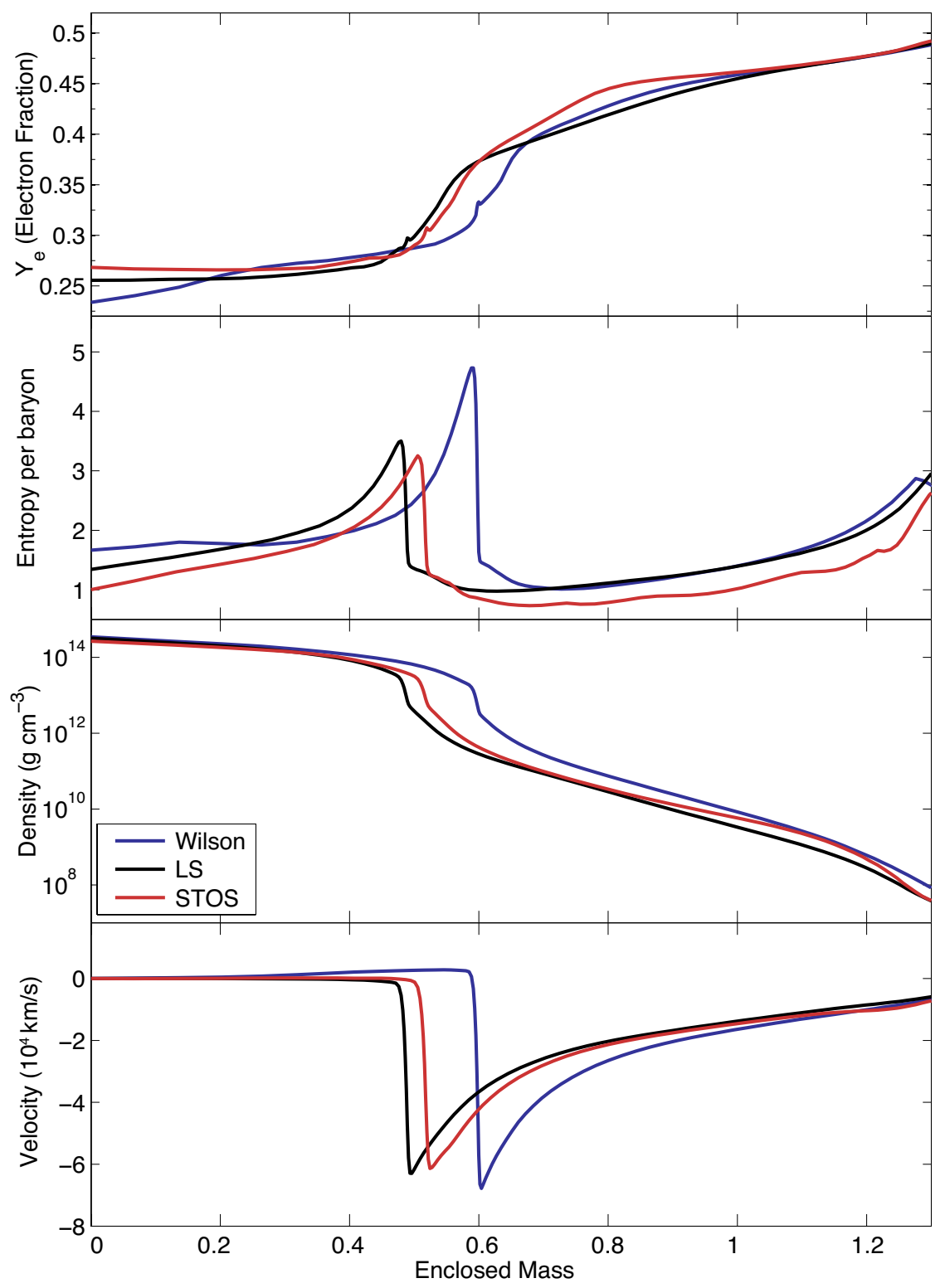

Figure 4: Effects of the equation of state at bounce for a $15 M_{\odot}$ progenitor. The black, red and blue lines illustrate results from simulations using the EoS of Lattimer and Swesty [13], Shen et al. [14] and Wilson and Mathews [15], respectively.

consistent with those simulation were used here, those from Bruenn [3] with the LMSH nuclear electron prescription. Figure 4 shows the conditions at bounce for the three EoS as a function of enclosed mass. The Wilson EoS results in a much larger homologous core $\left(0.58 M_{\odot}\right)$ than either STOS $\left(0.52 M_{\odot}\right)$ or LS $\left(0.48 M_{\odot}\right)$ cases. For much of the collapse, $Y_{e}$ for the Wilson cases is higher than the others, resulting in the larger core. At bounce, this effect can still be seen from 0.2-0.5 solar masses, though a sharp gradient in $Y_{e}$ develops in the Wilson EoS case over the last few ms before bounce in the regions with density above $2 \times 10^{14} \mathrm{~g} \mathrm{~cm}^{-3}$, where the pion contribution be- 


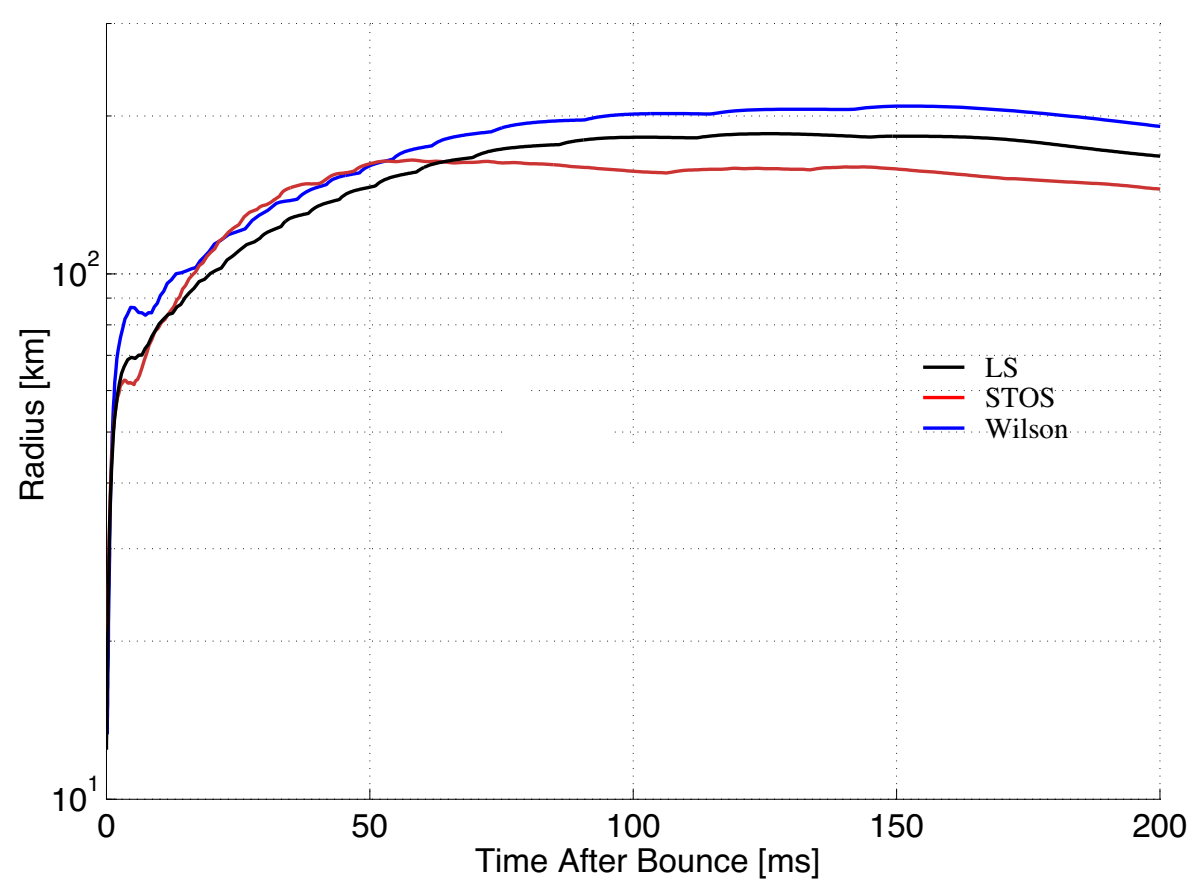

Figure 5: Impact of equation of state variations on the radius of the supernova shock as a function of time. The black, red and blue lines illustrate results from simulations using the EoS of Lattimer and Swesty [13], Shen et al. [14] and Wilson and Mathews [15], respectively.

comes significant. This decrease in the $Y_{e}$ is consistent with previous results [19] and is caused by the impact of the pions on the free nucleon chemical potentials. The differences in $Y_{e}$ at lower density between the three EoSs result from differences in the composition like those shown in Fig. 3. For example, the noticeable bump in $Y_{e}$ around $0.8 M_{\odot}$ in the STOS case coincides with largest difference in heavy nucleus mass fractions between the STOS and LS EoS. Likewise, the generally lower heavy nucleus mass fraction of the Wilson EoS results in less electron capture consistently throughout the star.

Figure 5 shows the time history of the shock over $200 \mathrm{~ms}$ after bounce. Note, the small scale noise seen beyond $10 \mathrm{~ms}$ in Figure 5 result from the determination of the location of the shock in Agile-Boltztran. In simulations with the $\operatorname{LS} \operatorname{EoS}\left(\kappa_{s}=180 \mathrm{MeV}\right)$, the shock is launched, and reaches approximately $189 \mathrm{~km}$ (enclosed mass of $1.38 M_{\odot}$ ) from the central core before stalling $130 \mathrm{~ms}$ after bounce. In contrast, the shock in simulations using the STOS only reaches a distance of approximately $165 \mathrm{~km}\left(1.32 M_{\odot}\right)$ before stalling. This maximum also occurs much sooner (60 ms after bounce) than in the LS EoS model. With $\kappa_{s}=180 \mathrm{MeV}$, LS EoS is a relatively soft EoS and, as a consequence, stores a larger amount of energy in the PNS than a stiffer EoS, allowing the shock to travel further out before stalling due to energy loses from neutrino emission and the disassociation of heavy nuclei. The STOS EoS, with $\kappa_{s}=280 \mathrm{MeV}$, is stiffer than the LS EoS, so while the initial pressures are higher and thus the early progress of the shock more rapid, less energy is stored in compression of the PNS. As a result the shock can not travel as far out from the central core before stalling. The shock in simulations with the Wilson EoS travels out to a 
distance of approximately $209 \mathrm{~km}\left(1.44 M_{\odot}\right)$ before stall, $\sim 20 \mathrm{~km}$ further than the LS EoS case, and $\sim 44 \mathrm{~km}$ further than the STOS EoS. Even though the Wilson EoS, with $\kappa_{s}=200 \mathrm{MeV}$, is nominally stiffer than the LS EoS, it provides more energy to the shock than the LS EoS does, likely as a consequence of both the high density pion contribution and the larger initial size of the PNS. Even $200 \mathrm{~ms}$ after bounce, by which time convective instabilities will have developed in the neutrino heated region, the shock's location, which marks the outer boundary of this unstable region, exhibits a significant $(\sim 20 \%)$ variation as a result of the EoS. This is a clear indication that the EoS is important to the physics of core collapse and bounce and its impact can extend even to times well after bounce.

\section{Conclusion}

While a full understanding of the role of nuclear physics in core-collapse supernovae awaits a better understanding of the supernova mechanism and the timescales on which it operates, it is clear that both nuclear interactions with electrons and neutrinos and the nuclear equation of state have significant impact on core collapse. As a result of differences in the nuclear composition, variations in the EoS produce nearly 20\% differences in the initial PNS mass, as well as differences in the electron fraction, density, and velocity ahead of the shock that persist for more than $100 \mathrm{~ms}$. When one considers the behavior of the supernova shock, these effects due to the EoS extend to at least $200 \mathrm{~ms}$ after bounce, well into the epoch where multi-dimensional effects begin to dominate the supernova evolution. Clearly, there is a continued need to improve our understanding of the supranuclear EoS which is responsible for launching the bounce shock. In addition, our results show a need to better understand the subnuclear composition. This later point is echoed in recent work on the impact on PNS cooling of the abundances of $\mathrm{H}$ and $\mathrm{He}$ isotopes at densities

$>10^{13} \mathrm{~g} \mathrm{~cm}^{-3}[24,25]$. Convolved with the neutrino-matter opacities, these mass fractions can have as large an impact as the supranuclear regime and therefore also warrant further study.

\section{Acknowledgments}

The authors have greatly benefited from discussions with K. Langanke, G. Martinez-Pindeo, A. Juodogalvis, H.-T. Janka, B. Müller, M. Meixner and G. Mathews. This work was supported by the NSF PetaApps program under grant numbers OCI-0749242, OCI-0749248, and OCI-0749204, by NASA under grant 07-ATFP07-0011, and by the U. S. Department of Energy under Contract No. DE-AC05-00OR22725 at Oak Ridge National Laboratory. The simulations described here were performed at the National Center for Computational Sciences at ORNL.

\section{References}

[1] H. A. Bethe, G. E. Brown, J. Applegate, and J. M. Lattimer, Nucl. Phys. A 324, 487 (1979).

[2] G. M. Fuller, W. A. Fowler, and M. J. Newman, Astrophys. J. 293, 1 (1985).

[3] S. W. Bruenn, Astrophys. J. Suppl. 58, 771 (1985). 
[4] K. Langanke, G. Martínez-Pinedo, J. M. Sampaio, D. J. Dean, W. R. Hix, O. E. Messer, A. Mezzacappa, M. Liebendörfer, H.-T. Janka, and M. Rampp, Phys. Rev. Lett. 90, 241102 (2003).

[5] W. R. Hix, O. E. B. Messer, A. Mezzacappa, M. Liebendoerfer, J. M. Sampaio, K. Langanke, D. J. Dean, and G. Martinez-Pinedo, Phys. Rev. Lett. 91, 201102 (2003).

[6] K. Langanke and G. Martínez-Pinedo, Nucl. Phys. A 673, 481 (2000).

[7] K. Langanke, E. Kolbe, and D. J. Dean, Phys. Rev. C 63, 032801 (2001).

[8] M. Liebendörfer, O. E. B. Messer, A. Mezzacappa, S. W. Bruenn, C. Y. Cardall, and F.-K. Thielemann, Astrophys. J. Suppl. 150, 263 (2004).

[9] A. Juodagalvis, K. Langanke, G. Martinez-Pinedo, W. R. Hix, D. J. Dean, and J. M. Sampaio, Nucl. Phys. A 747, 87 (2005).

[10] K. Langanke, G. Martínez-Pinedo, B. Müller, H.-T. Janka, A. Marek, W. R. Hix, A. Juodagalvis, and J. M. Sampaio, Physical Review Letters 100, 011101 (2008).

[11] S. W. Bruenn and W. C. Haxton, Astrophys. J. 376, 678 (1991).

[12] D. G. Ravenhall, C. J. Pethick, and J. R. Wilson, Phys. Rev. Lett. 50, 2066 (1983).

[13] J. Lattimer and F. D. Swesty, Nucl. Phys. A 535, 331 (1991).

[14] H. Shen, H. Toki, K. Oyamatsu, and K. Sumiyoshi, Prog. Theor. Phys. 100, 1013 (1998).

[15] J. R. Wilson and G. J. Mathews, Relativistic Numerical Hydrodynamics, Cambridge Monographs on Mathematical Physics (Cambridge University Press, Cambridge, 2003).

[16] D. Q. Lamb, J. M. Lattimer, C. J. Pethick, and D. G. Ravenhall, Phys. Rev. Lett. 41, 1623 (1978).

[17] K. Oyamatsu, Nucl. Phys. A 561, 431 (1993).

[18] R. L. Bowers and J. R. Wilson, Astrophys. J. Suppl. 50, 115 (1982).

[19] R. W. Mayle and J. R. Wilson, in Supernovae, edited by S. E. Woosley (Springer, New York, 1991), p. 333.

[20] T. L. McAbee and J. R. Wilson, Nucl. Phys. A 576, 626 (1994).

[21] E. Baron, J. Cooperstein, and S. Kahana, Phys. Rev. Lett. 55, 126 (1985).

[22] H. Muther, M. Prakash, and T. L. Ainsworth, Phys. Lett. B 199, 469 (1987).

[23] B. Friedman, V. R. Pandharipande, and Q. N. Usmani, Nucl. Phys. A 372, 483 (1981).

[24] A. Arcones, G. Martínez-Pinedo, E. O’Connor, A. Schwenk, H.-T. Janka, C. J. Horowitz, and K. Langanke, Phys. Rev. C 78, 015806 (2008).

[25] K. Sumiyoshi and G. Roepke, Phys. Rev. C 77, 055804 (2008). 